\title{
A Note on the Hyperpnoic Action of Ammonium Chloride in Cats.
}

\author{
By \\ TAISUKE SUZUKI. \\ (鉿木 退 輔) \\ (From the Plysiological Laboratory of Prof. Ir. Satalie, \\ Tohoku Imperial University, Sendai.)
}

Working with the hyperglycaemic action of ammonium chloriche Morita $a^{12}$ came to notice the absence of the marked acceleration of breathing when the salt was administered to cats, the splanchnic nerves of which had been interferred with bilaterally.

I have now undertaken to develop this question put forward by him by applying the salt to cats, whose splanchnic nerves on one side were divided and the suprarenal gland on the other side was removed. And the repetition of the experiments quoted in his communication was tried in the first place with results much similar to his. Further some unilaterally splanchnectomized cats with extirpating the suprarenal body have been also experimented on. All the data will be summarized in the following table, a protocol from the series of experiments on cats, whose splanchnic nerves on one side and the gland on the other had been interferred with.

Adult cats of good nutrition were employed. They were fed with a mixed diet (rice (mainly), fish, potato, radish and other vegetables cooked together with a little table salt and soy) for a few weeks at least before the experiment or operation. The sex was at random.

A mmonium chloride (Mer ck) was made in 4 mol solution warmed to about $38^{\circ}$ and injected hypodermically in the hind limbs. Since a smaller dose of ammonium chloride than $0.7 \mathrm{grm}$. per kilo of body weight was often found insufficient to bring about a quickening of respiration and general excitation in normal cats, it was given in a dose of $0.7 \mathrm{grm}$. per kilo through-

1) Sachi. Morita, Tohoku J. Exp. Med., 1922, 3, 410. 


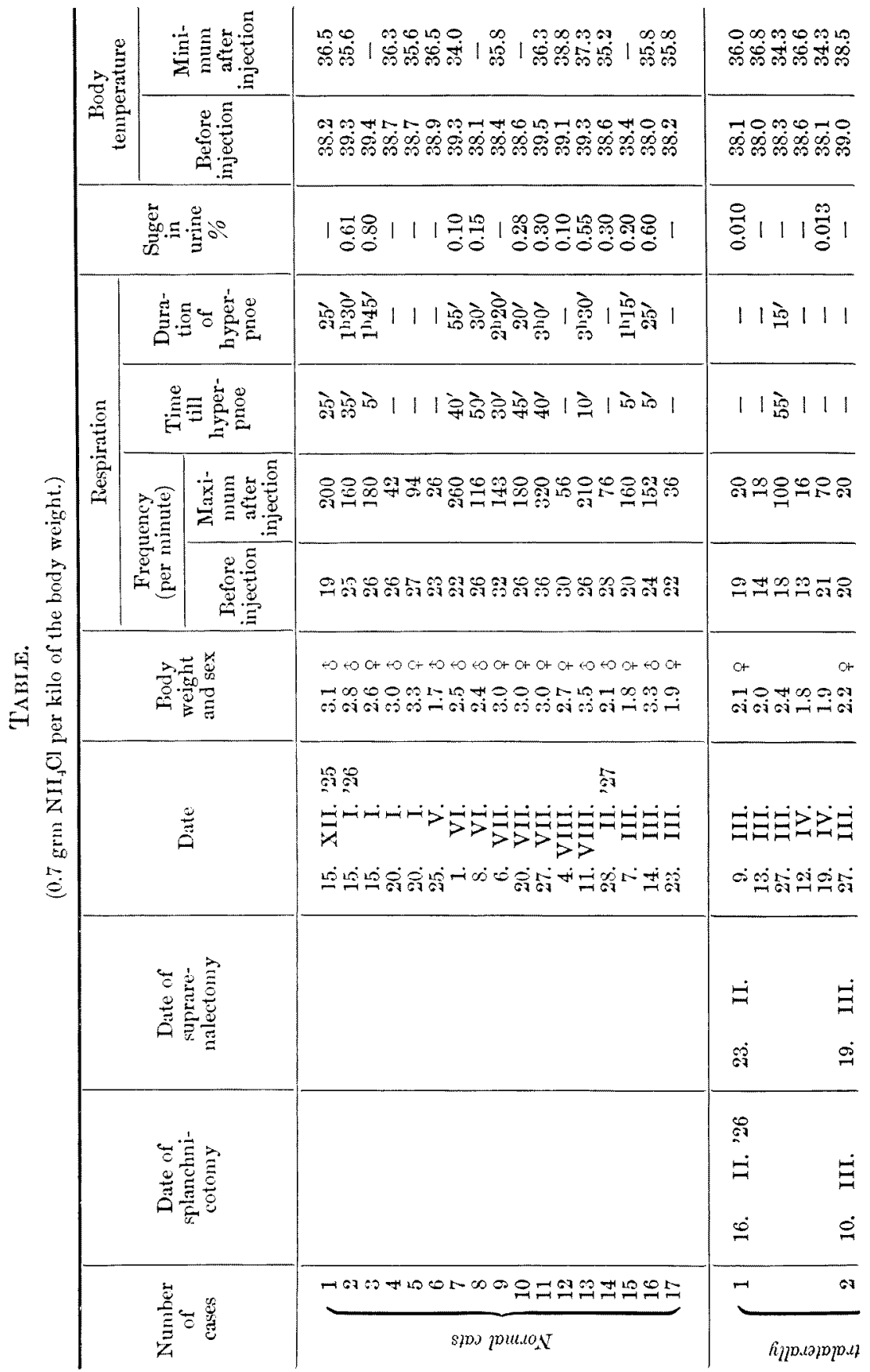




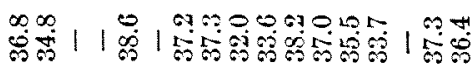

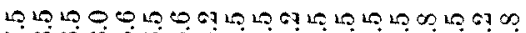
16

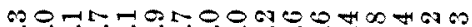

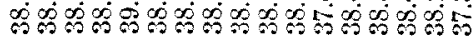

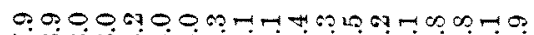
150\%

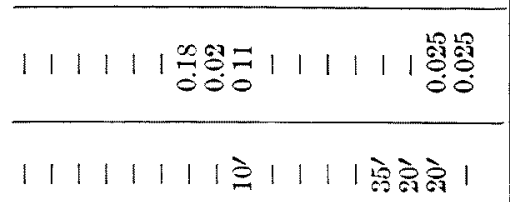

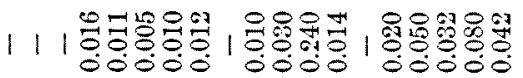

11111111111120

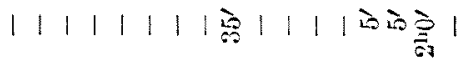

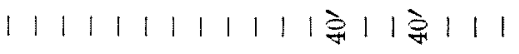

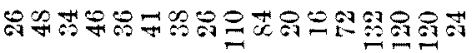

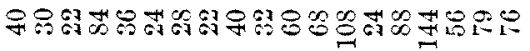

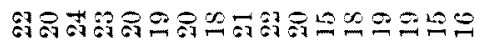

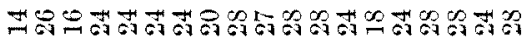

sos to to

$\mathrm{Cr}+\mathrm{CH}$

6)

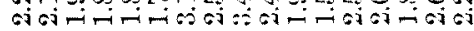

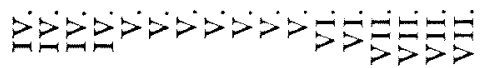

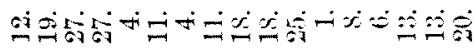

\begin{tabular}{|c|c|c|c|c|}
\hline$D$ & $=$ & 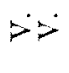 & $\Rightarrow$ & $\sum^{2}$ \\
\hline$\dot{\pi}$ & 51 & 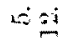 & ai & $1 \div$ \\
\hline$\Xi$ & $\Xi$ & $=$ & 75 & 5 \\
\hline$\stackrel{\circ}{\circ}$ & $\stackrel{\theta}{2}$ & $80^{\circ}$ & $\approx 0$ & $\stackrel{5}{8}$ \\
\hline 40 & 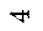 & $\mathrm{kSO}$ & $1-\infty$ & $\sigma$ \\
\hline
\end{tabular}

sppo pazzucopopouasd.edns

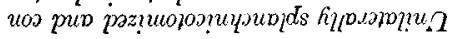

Ctct to to toot loot to to

OमN

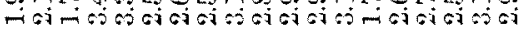

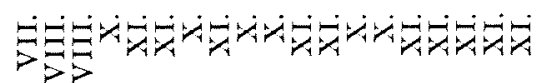

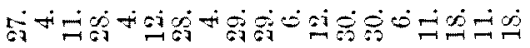

員

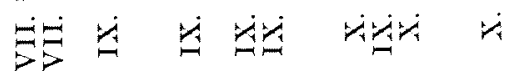

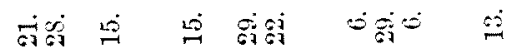

$-640100000$

spor paznuoponuynoqds fllpalol!g 


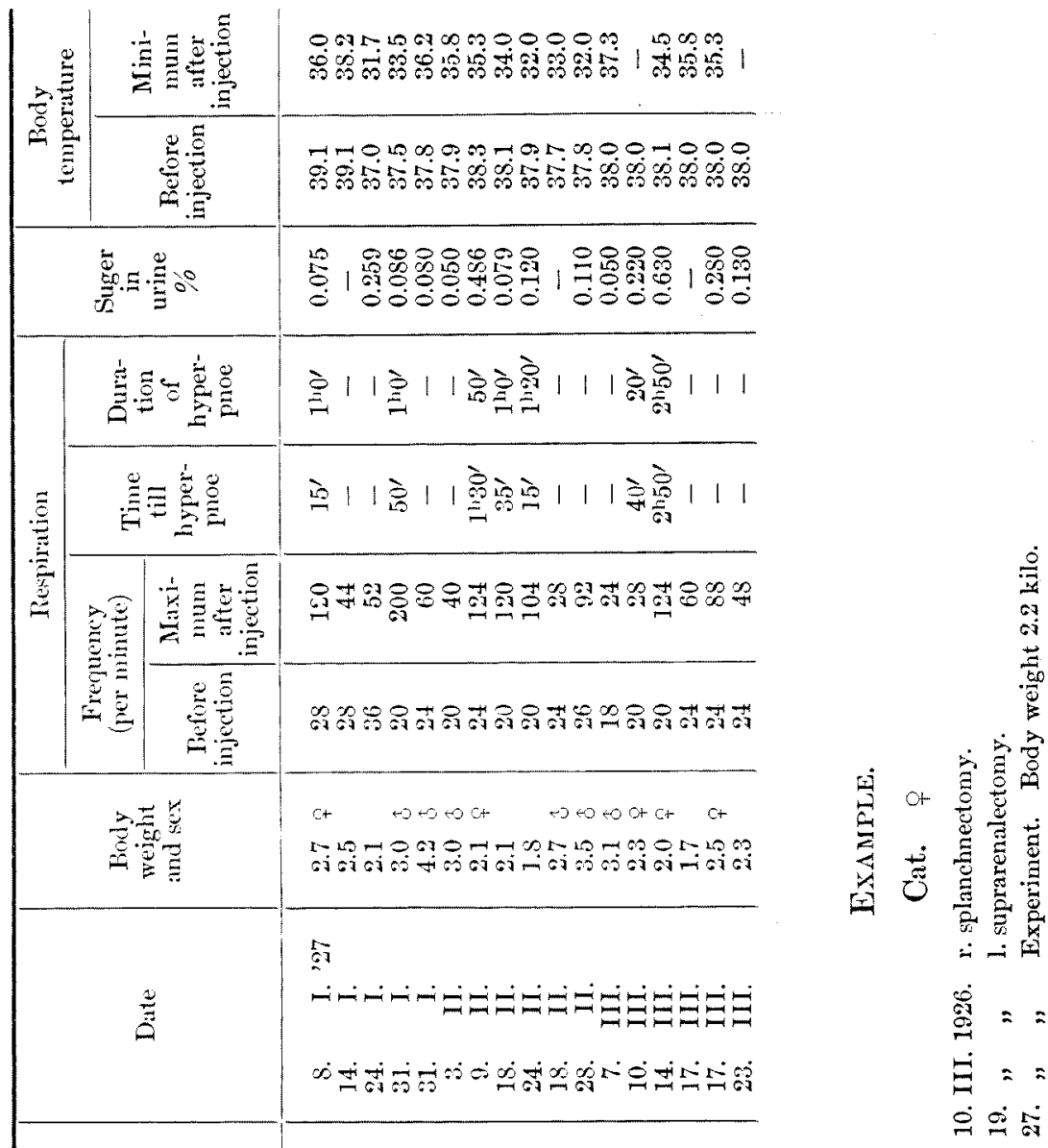

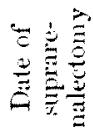

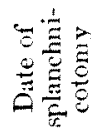

方

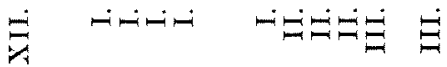

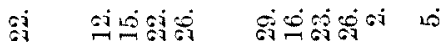

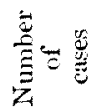

$-60041000000$

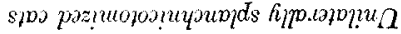




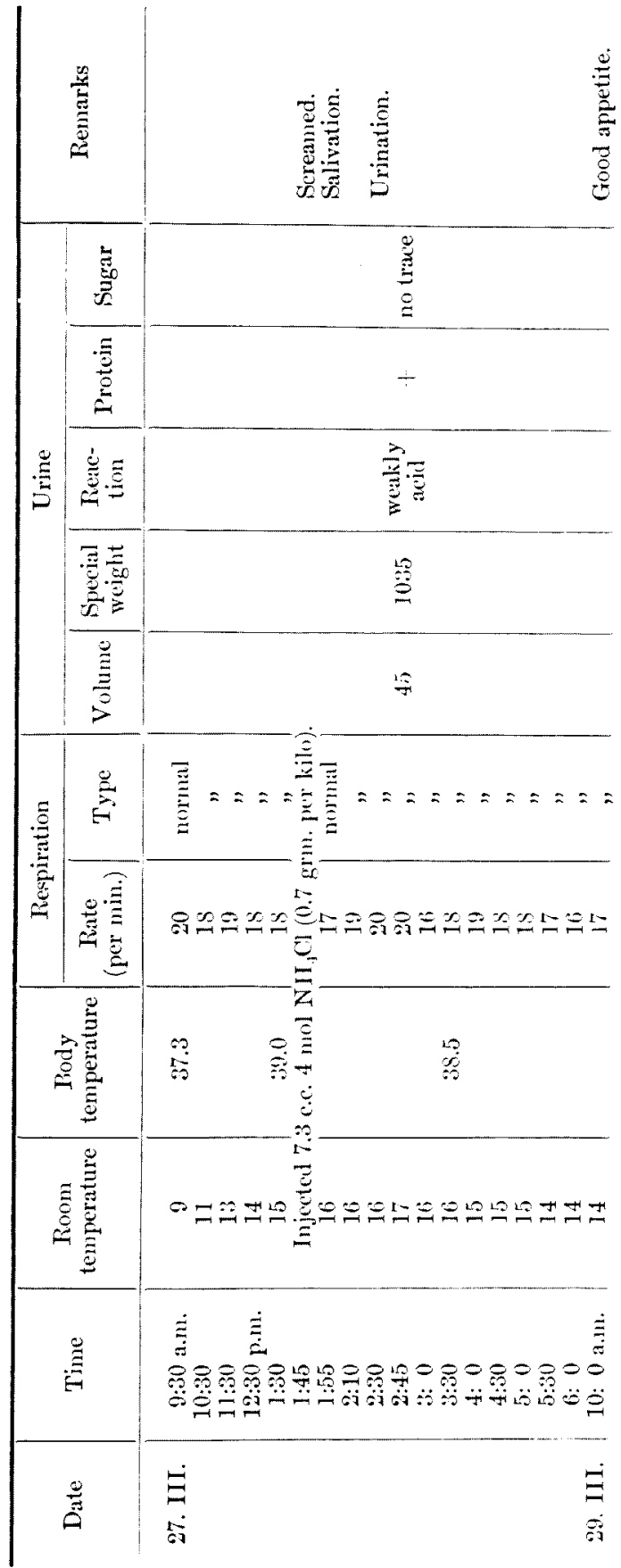

out all the series of experiments. The dose was proved sufficient as a rule to manifest its action.

Suprarenalectomy and splanchnicotomy were carried out extraperitoneally by the lumbar route some weeks prior to the experiment.

Sugar in urine was measured by Bertrand.

\section{Results.}

1. Experiments on normal cats.

Soon after the hypodermical injection of the salt solution the cat screamed, behaved itself as if it would bite somewhere in the region where the injection was made, and rolled over violently. The pupils widened ad maximum, and the nictating membranes drew back. Before long the animal became quiet, while salivation, vomiting, defaecation and micturition took place for about half an hour after the injection. 
In almost all instances the breathing became very rapid immediately after the injection. The maximum in the rate was noted as 100-150 a minute or more which occurred within about half an hour after the injection. Inspiratory movements of the wings of the nose and convulsion-like breathing were also often observed. In a few cases a great acceleration in the breathing did not occur, but it was of convulsion-like type. The hyperpnoic interval lasted from half an hour to three hours or longer.

After the exciting period had passed away, the animal lay down calmly on its side and fell asleep. For a few days after the experiment the animal looked weak and had no good appetite. The body temperature descended invariably during the poisoning. Sugar and protein were found almost always in the urine.

2. Experiments on cats, whose splanchnic nerves on one side and suprarenal body on the other were interferred with.

For this set of experiments nine cats were prepared by unilateral splanchnicotomy and contralateral suprarenalectomy, and with each cat some experiments were carried out as a rule.

Ammonium chloride in a dose of $0.7 \mathrm{grm}$. per kilo was capable of producing in this sort of cat screaming, salivation, vomiting, defaecation and micturition as in normal animals, but they seemed of a somewhat minor degree. In some cases the hyperpnoea was remarkable, but its duration was distinctly shorter than in the normal individuals. An acceleration such as exceeding 150 a minute was never noticed in this set of experiments. And in the remaining cases, that is in the majority, the respiration, both in rate and type, remained absolutely or nearly unaltered notwithstanding the manifestation of the other symptoms above related. In one and the same animal the hyperpnoea occurred occassionally, while on other days no alteration took place in breathing; why it was so, I an not able to make any suggestion. The body temperature also decreased; sugar was only seldom discovered in the urine and only in a very small quantity. The recovery of the animals from the poisoning was seemingly somewhat sooner in comparison with the normal ones.

3. Experiments on bilaterally splanchnicotomized cats.

The number of cats employed for this set of experiments was ten, and the half of them was utilized two or three times. Of the poisoning symptoms, their severity and the velocity of recovery from poisoning it was difficult to distinguish this set of experiments from that quoted in the above paragraphs. The breathing became rapid on the injection of ammonium chloride in rare cases. Even when the acceleration set in, it lasted only 
for a while. Namely in a great number of instances the respiration was not materially influenced by administration of the salt. Of the frequency of non-occurrence of rapid breathing on the injection of $\mathrm{NH}_{4} \mathrm{Cl}$ this set of experiments is seemingly a little superior to that of cats which were unilaterally suprarenalectomized instead of cutting the splanchnic nerves, but it may not be justifiable to state this definitely because of the small number experiments. Of the body temperature and sugar in the urine similar results to those in the above section must be repeated here.

4. Experiments on unilaterally splanchnicotomized cats.

The intensity of the excitements following the administration of the salt in the same quantity was in the unilaterally splanchnicotomized cats rather similar to the normal individuals. In relation to the respiratory movements the results of this set of experiments were intermediate, that is the acceleration occurred more frequently as in the bilaterally splanchnicotomized ones or the unilaterally splanchnicotomized and contralaterally suprarenalectomized, but not so commonly as in the normal cats. In about one third of the cases the rapid breathing as over 100 a minute was observed, and it continued one hour or longer. Even when no rapid rate was counted, the breathing was usually laboured. Glycosuria and albuminuria were also detected in almost all cases. The body temperature descended.

Now by again epitomizing the data given in the above table the following small table is prepared. The classification of the intensity of acceleration in three degrees, strong, intermediate and weak or none, is certainly artificial ; there are naturally no boundaries between the degrees.

Acceleration of the frecuency of respiration

\begin{tabular}{l|c|c|c|c}
\hline & $\begin{array}{c}\text { Total } \\
\text { number } \\
\text { of }\end{array}$ & \multicolumn{3}{|c}{ Degree } \\
\cline { 3 - 5 } & cases & Strong & $\begin{array}{c}\text { Inter- } \\
\text { mediate }\end{array}$ & $\begin{array}{c}\text { Weak or } \\
\text { none }\end{array}$ \\
\hline $\begin{array}{c}\text { Normal cats } \\
\text { Cnilaterally splanchnicotomized } \\
\text { and contralaterally suprarena- }\end{array}$ & 17 & $11(65 \%)$ & $4(23 \%)$ & $2(12 \%)$ \\
$\begin{array}{c}\text { Bectomized cats } \\
\text { Bilaterally splanchnicotomized } \\
\text { cats }\end{array}$ & 23 & $5(22 \%)$ & $6(26 \%)$ & $12(52 \%)$ \\
Unilaterally splanchnicotomized \\
cats
\end{tabular}

\section{SUMMARY.}

As is readily seen by examining the above small table, the results with the intact cats and the bilaterally splanchnicotomized fell into the same line 
with those of Morita ; that is, the normal cats respond against the subcutaneous injection of ammonium chloride with an increase in the rate of breathing, remarkable or moderate; a definite acceleration was unperceived in a few individuals only. On the other hand, the double splanchnicotomy was capable of abolishing this reaction to a great extent, namely in over half of the cases an unmistakable increase in the respiration rhythm failed to occur.

The events caused by the salt in the cats, whose suprarenal body on one side was removed and the splanchnic nerves on the other divided, did not differ very materially from those with the bilaterally splanchnicotomized, but plainly differed from those with the unilaterally splanchnicotomized but not suprarenalectomized ones.

In relation to the hyperpnoic action of ammonium chloride the suprarenalectomy appears, thus, to have a similar bearing as to the splanchnicotomy, though it may be somewhat inferior.

I practised great care not to interfere with the splanchnic fibres which do not destinate in the suprarenal gland, but it is a matter of great difficulty to speak with confidence of the integrity of all or nearly all of such fibres. We are acquainted with the facts, which indicate that the remoral of the suprarenal gland may run a risk of damaging the splanchnic nerves in an unexpected nanner. The reports, for example, that the change in the blood pressure curre caused by sensory stimulation or stimulation of the peripheral end of the splanchnic nerve disappears after the extirpation of both the suprarenal glands, were annibilated by subsequent researches. The fuilure of the change was really caused by injury of the splanchnic nerves and not by the removal of the glands themselves.

On perusal of the literature it may be justifiable to assume that the rôle of the suprarenal gland in the hyperpnoic action of ammoniurn chloride cannot be explained by the orersecretion of epinephrine. It is open to further investigation. 